\title{
Vibrational-state and isotope dependence of high-order harmonic generation in water molecules
}

\author{
Mirjam Falge, ${ }^{1}$ Volker Engel, ${ }^{1}$ and Manfred Lein ${ }^{2}$ \\ ${ }^{1}$ Universität Würzburg, Institut für Physikalische Chemie, Am Hubland, D-97074 Würzburg, Germany \\ ${ }^{2}$ Centre for Quantum Engineering and Space-Time Research (QUEST) and Institut für Theoretische Physik, Leibniz Universität Hannover, \\ Appelstraße 2, D-30167 Hannover, Germany \\ (Received 4 December 2009; published 16 February 2010)
}

\begin{abstract}
We report calculations on high-order harmonic generation in water molecules. Spectra are determined for various initial vibrational states of $\mathrm{H}_{2} \mathrm{O}$ and its isotope $\mathrm{D}_{2} \mathrm{O}$. It is demonstrated that the ratio of the spectra for $\mathrm{D}_{2} \mathrm{O}$ and $\mathrm{H}_{2} \mathrm{O}$ is close to unity when the initial state is the vibronic ground state, indicating that nuclear dynamics is of minor importance. For vibrationally excited initial states, the high-harmonic intensities show a clear dependence on both the initial-state quantum number and the isotopic species.
\end{abstract}

DOI: 10.1103/PhysRevA.81.023412

PACS number(s): $33.80 . \mathrm{Rv}, 42.65 . \mathrm{Ky}$

\section{INTRODUCTION}

The interaction of high-intensity laser fields with atoms and molecules induces a number of nonlinear, nonperturbative processes. High-order harmonic generation (HHG) [1,2] is one of the most interesting of these phenomena, because it provides a source of coherent high-frequency radiation and attosecond pulses [3,4]. Traditionally, rare gases have served as the most widely used targets for HHG. In recent years, the interest in HHG from molecules has been increasing rapidly. The starting point of this development was the combination of HHG with experimental techniques for molecular alignment, leading to the observation that the harmonic intensity can depend strongly on the direction of alignment for $\mathrm{CS}_{2}, \mathrm{CO}_{2}$, $\mathrm{N}_{2}$, and $\mathrm{O}_{2}$ [5-7]. Theoretical modeling showed that for simple cases such as $\mathrm{H}_{2}{ }^{+}$and $\mathrm{H}_{2}$, the presence of two centers causes a distinct signature of two-center interference in the HHG spectra and in the orientation dependence of HHG [8,9]. For the $\mathrm{CO}_{2}$ molecule, experimental observations in agreement with the two-center interference picture were presented by different groups [10,11]. However, a more detailed analysis [12] indicates that not only the outermost electron orbital but also lower lying orbitals contribute to the observed effects in $\mathrm{CO}_{2}$. Recent experiments on $\mathrm{N}_{2}$ support contributions of multiple orbitals [13] as well. Alignment dependence of HHG has also been demonstrated in the polyatomic molecules acetylene and allene [14].

The fact that HHG is sensitive to the molecular electronic structure has been exploited in the orbital tomography scheme for reconstructing molecular orbitals from measured harmonic spectra [15]. Furthermore, the electronic-structure dependence implies that the harmonic yield is also sensitive to changes in the atomic positions as they occur in molecular vibrational motion and in chemical reactions. Harmonic spectra have thus been used as a probe signal in pump-probe experiments on $\mathrm{SF}_{6}$ and $\mathrm{N}_{2} \mathrm{O}_{4}$ to observe both small and large amplitude vibrational wave-packet motion $[16,17]$.

In molecular systems with a short vibrational period, significant nuclear motion may take place during the HHG process itself. The finite duration of the HHG process can easily be estimated by invoking the most popular qualitative explanation of HHG: according to the semiclassical three-step model [18], the laser field first ionizes the atomic or molecular system, subsequently accelerates the free electron, and finally drives it back to the parent ion. Recombination of the returning electron leads to the emission of a high-frequency photon. From this model, it is apparent that any nuclear motion in the time interval between ionization and recombination will have a significant effect on the efficiency. Differences in harmonic yields comparing the hydrogen molecule $\mathrm{H}_{2}$ and its heavier isotope $\mathrm{D}_{2}$ have been predicted [19] and measured [20,21]. The heavier isotope generates harmonics more efficiently because of the slower nuclear motion. A similar isotope dependence has been observed in methane and deuterated methane [20].

In the present study, we investigate $\mathrm{HHG}$ in the water molecule. In particular, we are interested in a possible isotope effect arising when the $\mathrm{H}$ atoms are replaced by D atoms. HHG experiments with water microdroplets have been reported [22]. At the time, however, the main focus was on optimizing the generation efficiency, and isotope effects were not investigated. In the present work, we show that isotope effects are negligible as long as we study water molecules that are prepared in their vibrational ground state. We then consider initial states that are vibrationally excited. Such states have been examined, for example, in experiments on state-selective [23,24] and vibrationally mediated photodissociation $[25,26]$ of water and also theoretical studies on ladder climbing in $\mathrm{H}_{2} \mathrm{O}$ via infrared excitation [27].

From a theoretical point of view, it is clear that an exact numerical quantum mechanical description of the $\mathrm{HHG}$ process in $\mathrm{H}_{2} \mathrm{O}$, including both electronic and nuclear motion, is presently out of scope. Full-dimensional calculations have been achieved in the case of $\mathrm{H}_{2}{ }^{+}$with the direction of the molecular axis restricted to the laser polarization axis [28]. In most cases, however, reduced dimensional models have been used to study coupled electronic and nuclear motion in strong laser fields [29-33].

In the present work, we apply an extension of the strongfield approximation (SFA) for HHG, introduced for atoms by Lewenstein et al. in 1994 [34]. The nuclear dynamics enters the model via a time-correlation function [19]. It accounts for the motion of a nuclear wave packet launched in the molecular ion by the ionization step. This method has previously been used to describe the isotope effects in HHG from $\mathrm{H}_{2}$ and $\mathrm{D}_{2}$. If the electronic bound-continuum transition matrix elements that enter the SFA depend only weakly on the nuclear positions, one arrives at a simpler SFA model, where the nuclear motion is 
contained in the autocorrelation function of the nuclear wave packet, independent of the electronic structure [19]. Such a case is realized in the water molecule. Recently, the time evolution of the autocorrelation function has been calculated for a broad range of diatomic and polyatomic molecules (excluding $\mathrm{H}_{2} \mathrm{O}$ ) [35]. This study showed that the influence of nuclear motion on HHG is not limited to bound protons, but it is clearly present in molecules such as $\mathrm{NO}$ or $\mathrm{NO}_{2}$.

The article is organized as follows: In Sec. II, we summarize the working equations for the SFA including the nuclear degrees of freedom and describe the model for the water molecule. Harmonic spectra for different isotopes and initial vibrational states are presented in Sec. III, which also contains a short conclusion.

\section{THEORY}

\section{A. Strong-field approximation for molecules}

Even at the level of the single-atom or single-molecule response, the exact calculation of harmonic spectra is possible only in very simple cases such as the hydrogen atom. Therefore, in general one is forced to employ one or the other approximation. Here, we adopt the SFA for HHG, which was introduced by Lewenstein et al. [34] to study HHG from atoms. Later, the model was extended to molecules, including the nuclear motion $[19,36]$, and applied to $\mathrm{HHG}$ in $\mathrm{H}_{2}$ and its isotopes. For the $j$ th vibrational state of the neutral molecule as initial state, the harmonic spectrum is obtained as (atomic units are used throughout)

$$
S_{j}(\omega)=\omega^{2}\left|\int_{0}^{T} V_{D, j}(t) e^{i \omega t} d t\right|^{2},
$$

where $T$ denotes the pulse length and $\omega$ denotes the laser frequency. Here, the dipole velocity is

$$
\begin{aligned}
V_{D, j}(t)= & \frac{2 i}{(2 \pi)^{3}} \int_{-\infty}^{t}\left[\frac{2 \pi}{\epsilon+i\left(t-t^{\prime}\right)}\right]^{3 / 2} c_{j}\left(t-t^{\prime}\right) v^{*}\left(k_{1}\right) \\
& \times d\left(k_{2}\right) E\left(t^{\prime}\right) e^{-i \tilde{S}_{j}\left(t, t^{\prime}\right)} d t^{\prime}+\text { c.c. },
\end{aligned}
$$

where $E(t)$ denotes the electric field pointing in the $z$ direction. The interval $\left(t-t^{\prime}\right)$ corresponds to the travel time of the electron, which passes between the ionization $\left(t^{\prime}\right)$ and recombination $(t)$ processes. The parameter $\epsilon$, which prevents a singularity at $t=t^{\prime}$, was set to a value of $\epsilon=1$ a.u. in our calculations. The dipole velocity contains contributions from the electronic as well as from the nuclear degrees of freedom, which are identified in what follows. The electronic dipole and dipole-velocity matrix elements $d(k)$ and $v(k)$ are defined as

$$
\begin{aligned}
d(k) & =-\int d^{3} r e^{-i k z} z \varphi(\mathbf{r}), \\
v(k) & =-k \int d^{3} r e^{-i k z} \varphi(\mathbf{r}) .
\end{aligned}
$$

The first ionization band of water occurs above $I_{p}=0.46$ a.u. $(12.6 \mathrm{eV})$ [37] and corresponds to the removal of an electron from the ${ }^{1} b$ molecular orbital, which coincides with the $p_{z}$ orbital of atomic oxygen [38]. We assume that $\mathrm{H}_{2} \mathrm{O}$ is oriented such that the $p_{z}$ orbital (which is orthogonal to the plane spanned by the nuclei) points along the laser polarization vector. Thus, we take the bound-state electronic wave functions as

$$
\varphi(\mathbf{r})=N z e^{-a r},
$$

in evaluating the matrix elements. The values of $a=2.275$ a.u. and $N=\sqrt{\left(a^{5}\right) / \pi}$ are taken from Ref. [39]. The emerging electron is described by a plane wave with momentum $\mathbf{k}=k \mathbf{e}_{z}$. This allows for straightforward evaluation of the matrix elements as

$$
\begin{gathered}
d(k)=-32 \sqrt{\pi} \frac{a^{7 / 2}\left(a^{2}-5 k^{2}\right)}{\left(a^{2}+k^{2}\right)^{4}}, \\
v(k)=32 \sqrt{\pi} i \frac{a^{7 / 2} k^{2}}{\left(a^{2}+k^{2}\right)^{3}} .
\end{gathered}
$$

When the configuration is changed such that the orbital is no longer parallel to the laser polarization axis, it is expected that the HHG yield decreases smoothly, reaching (within our approximation) a value of zero at perpendicular orientation. The dipole moment and velocity $d\left(k_{2}\right), v\left(k_{1}\right)$ depend on the momenta in the presence of the external field, that is,

$$
k_{1}=p_{s}\left(t, t^{\prime}\right)+A(t), \quad k_{2}=p_{s}\left(t, t^{\prime}\right)+A\left(t^{\prime}\right),
$$

where

$$
\begin{gathered}
A(t)=-\int_{-\infty}^{t} E\left(t^{\prime}\right) d t^{\prime}, \\
p_{s}\left(t, t^{\prime}\right)=-\frac{1}{t-t^{\prime}} \int_{t^{\prime}}^{t} A\left(t^{\prime \prime}\right) d t^{\prime \prime} .
\end{gathered}
$$

During the motion of the electron in the continuum, it acquires a phase, which is

$$
\tilde{S}_{j}\left(t, t^{\prime}\right)=\int_{t^{\prime}}^{t}\left\{\frac{\left[p_{s}\left(t, t^{\prime}\right)+A\left(t^{\prime \prime}\right)\right]^{2}}{2}-E_{j}\right\} d t^{\prime \prime} .
$$

Here enters the energy $E_{j}$ of the vibrational eigenstate $\left|\psi_{j}\right\rangle$ of the neutral molecule. Finally, the nuclear dynamics is contained in the autocorrelation function, defined as

$$
c_{j}(\tau)=\left\langle\psi_{j}\left|U_{+}(\tau)\right| \psi_{j}\right\rangle,
$$

where the brackets denote integration over the nuclear degrees of freedom. The autocorrelation function involves the initial vibrational eigenstate, which evolves in time under the action of the propagator $U_{+}(\tau)$ in the ionic state. The time $\tau=t-t^{\prime}$ measures the travel time of the electron between ionization and recombination. In our approximation, the electronic matrix elements are independent of the positions of the nuclei. This is the reason for the factorized structure of the integrand in Eq. (2), where the function $c_{j}$ incorporates the effects of the nuclear motion and the ionization and recombination matrix elements incorporate the electronic structure of the molecule.

\section{B. Vibrational motion}

We treat the water molecule, taking two internal vibrational degrees of freedom $\left(x_{1}, x_{2}\right)$ into account. They correspond to the $\mathrm{H}-\mathrm{O}$ (or $\mathrm{D}-\mathrm{O}$ ) distances, whereas the bond angle is fixed to a value of $\gamma=104^{\circ}$. This value coincides with the bending angle in the electronic ground state. We ignore 
geometry changes in the angular degree of freedom that may occur upon ionization. This is a good approximation because the equilibrium angle in the $\left(\mathrm{H}_{2} \mathrm{O}\right)^{+}$ground state differs only slightly $\left(110^{\circ}[40]\right)$.

Within the light-heavy-light approximation, the center of mass is taken as the position of the $\mathrm{O}$ atom so that the employed nuclear Hamiltonians are as follows:

$$
H_{\mathrm{g} /+}=-\frac{1}{2 m}\left[\frac{\partial^{2}}{\partial x_{1}^{2}}+\frac{\partial^{2}}{\partial x_{2}^{2}}\right]+V_{\mathrm{g} /+}\left(x_{1}, x_{2}\right),
$$

where $m=m_{H}$ or $m=m_{D}$. The potentials $V_{\mathrm{g}}\left(x_{1}, x_{2}\right)$ and $V_{+}\left(x_{1}, x_{2}\right)$ are those corresponding to the neutral and ionic ground states, respectively, and are taken from Refs. [40,41], respectively. For simplicity, the upper potential is shifted such that the vertical energy difference at the equilibrium geometry of the neutral state coincides with the ionization potential $I_{p}$. We calculate the initial (neutral) vibrational wave functions $\psi_{j}\left(x_{1}, x_{2}\right)$ using imaginary-time propagation [42]. The autocorrelation functions are determined by real-time propagation. In both cases, we employ the split-operator technique [43].

\section{RESULTS}

\section{A. Harmonic spectra for $\mathrm{H}_{2} \mathrm{O}$ and $\mathrm{D}_{2} \mathrm{O}$}

In Fig. 1, we compare the harmonic spectra of $\mathrm{H}_{2} \mathrm{O}$ and $\mathrm{D}_{2} \mathrm{O}$, calculated for a laser wavelength of $800 \mathrm{~nm}$ and an intensity of $1.0 \times 10^{15} \mathrm{~W} / \mathrm{cm}^{2}$. For the pulse envelope, we use trapezoidal functions where the linearly increasing or decreasing parts are two optical cycles long and the constant middle part measures four cycles. The spectra show the typical plateau with a cutoff at high energies. The cutoff energy $3.17 U_{p}+I_{p}$ is calculated to be $E_{\text {cut }}=7.4$ a.u., which is in excellent agreement with the numerical results. Note that because of the plane-wave approximation for the matrix elements, the SFA is not expected to yield accurate results at low harmonic orders. The spectrum for the $\mathrm{D}_{2} \mathrm{O}$ isotope does not show a remarkable difference compared to the one obtained from $\mathrm{H}_{2} \mathrm{O}$. We have calculated the ratio of the integrated peak intensities and found that it is close to unity independent of the energy (not shown). This behavior is quite different

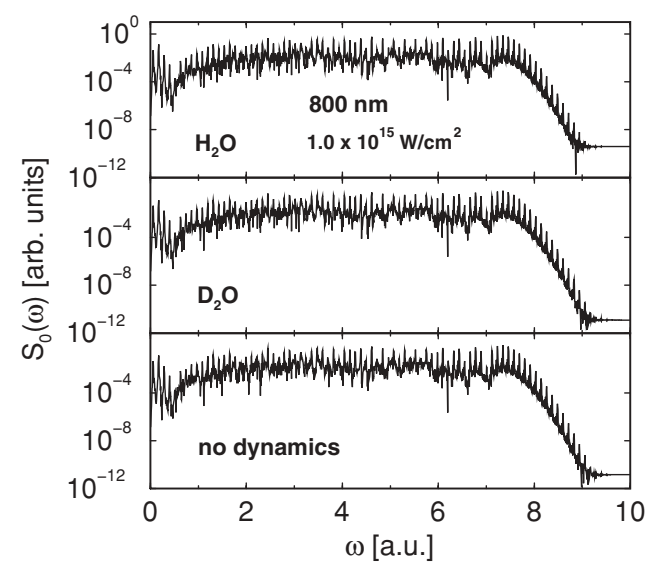

FIG. 1. Harmonic spectra of $\mathrm{H}_{2} \mathrm{O}$ and $\mathrm{D}_{2} \mathrm{O}$ calculated for a laser wavelength of $800 \mathrm{~nm}$ and an intensity of $10^{15} \mathrm{~W} / \mathrm{cm}^{2}$. The lower panel shows a spectrum for the case of frozen nuclear motion. from what is found for the $\mathrm{CD}_{4}$ and $\mathrm{CH}_{4}$ systems, where ratios up to 1.75 are found [20]. Similarly large numbers are predicted for the hydrogen molecule $[19,36]$. In the water molecule, the outermost electron does not participate in the $\mathrm{OH}$ bonding. As a consequence, the electronic structure does not change drastically upon ionization, so that the potential energy surfaces for the neutral and ionic ground states are quite similar in the vicinity of their equilibrium configurations. This is already evident from photoelectron spectra, which exhibit an intense (0)-(0) transition and a short vibrational progression [37]. Our calculations show that, for the relevant times, the time propagation in the ionic state proceeds mainly along the symmetric stretch coordinate, that is, along the symmetry line. This motion has a small amplitude as a result of the similar potential energy surfaces. It is found that the modulus of the autocorrelation function decays from its initial value $c_{0}(0)=1$ to a value of 0.66 in the first $5 \mathrm{fs}$. The relevant time interval for radiative recombination is in fact less than one optical cycle of the laser field (the latter corresponds to a time of $\sim 2.6 \mathrm{fs}$ ). The decay behavior in the case of $\mathrm{D}_{2} \mathrm{O}$ is very similar, and because this is the main difference entering into the calculation of the harmonic spectra, it is clear that the spectra are alike. [A minor difference is that the vibrational energy, being slightly different for the two isotopes, enters into the action; see Eq. (11).] Finally, we carry out a calculation, where the autocorrelation function is set to a value of $c_{0}(t)=\exp \left(-i t E_{+, 0}\right)$; that is, we replace the Hamiltonian $H_{+}$with the ionic vibrational ground state energy $E_{+, 0}$ so that the nuclear dynamics is frozen. The respective harmonic spectrum is displayed in the lower panel of Fig. 1. No major differences are found between this spectrum and the others, which include the dynamical effects. In Fig. 2, we compare spectra for the lower intensity, $5 \times 10^{14} \mathrm{~W} / \mathrm{cm}^{2}$, resulting in a cutoff at the lower energy, $E_{\text {cut }}=3.95$ a.u.

To conclude, the vibrational dynamics is of minor importance in harmonic generation from the water molecule and its isotope. This statement, however, relies on the initial condition that the neutral molecule is in its vibronic ground state. In the next subsection, HHG from excited vibrational states is discussed.

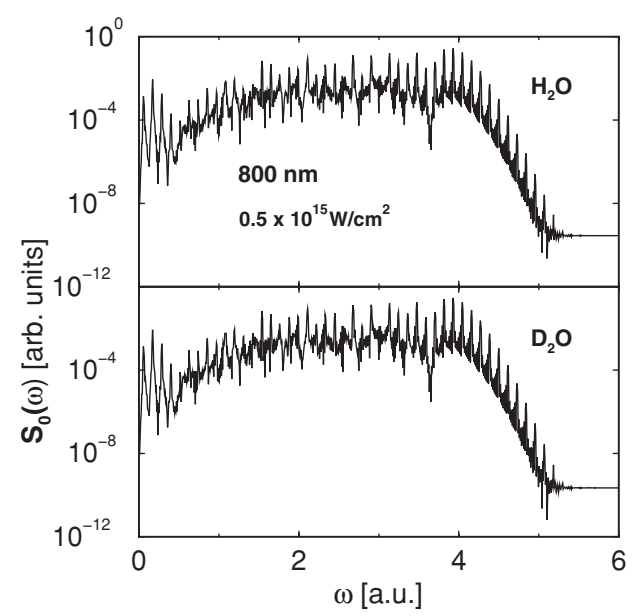

FIG. 2. Harmonic spectra of $\mathrm{H}_{2} \mathrm{O}$ (upper panel) and $\mathrm{D}_{2} \mathrm{O}$ (lower panel) calculated for a laser wavelength of $800 \mathrm{~nm}$ and an intensity of $0.5 \times 10^{15} \mathrm{~W} / \mathrm{cm}^{2}$. 


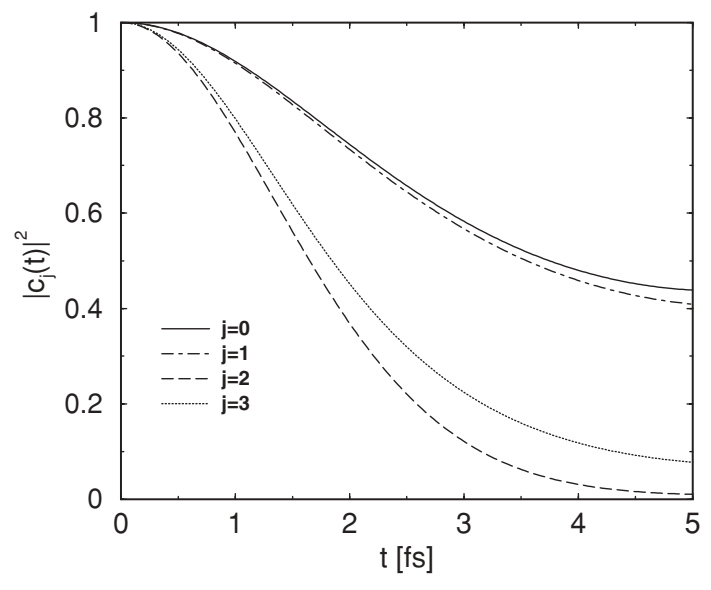

FIG. 3. Modulus squared of the autocorrelation functions for $\mathrm{H}_{2} \mathrm{O}$. Shown is the time dependence for different vibrational states with quantum numbers $j$, as indicated.

\section{B. Vibrational-state dependence of HHG}

From the discussion in Sec. III A, it emerges that if one starts in the vibronic ground state, the nuclear dynamics in the ionic state has hardly any influence on the harmonic spectrum. In what follows, we consider HHG for the case that the molecule is initially vibrationally excited. Because the nuclear dynamics enters through the autocorrelation functions into the calculation of the spectra, we first inspect the time dependence of these functions for selected values of the initial vibrational quantum number $j$. Figure 3 displays the modulus squared of $c_{j}(t)$ for the values $j=0,1,2,3$. While the functions originating from the time evolution of the two lowest vibrational states show a similar decay behavior, the decay is much faster for the higher excited initial states. The decay is determined by the wave-packet motion in coordinate space (i.e., the separation from the Franck-Condon region) and also in momentum space (corresponding to a coordinate dependent phase). The differences in extent and nodal structure of the various wave functions cause differences in the decay of the autocorrelation functions. To characterize the signature of this behavior, we regard specific high-order harmonic peaks. In Fig. 4, we show the ratios

$$
R_{j}\left(\omega_{n}\right)=\frac{S_{j}\left(\omega_{n}\right)}{S_{0}\left(\omega_{n}\right)}
$$

where $\omega_{n}$ is the frequency of the $n$th harmonic and the vibrational quantum number $j$ characterizes the degree of excitation in the neutral state. The figure exhibits curves calculated for $\mathrm{H}_{2} \mathrm{O}$ and $\mathrm{D}_{2} \mathrm{O}$ for $n=39$ and $n=69$, as indicated. In the calculation, we employ a wavelength of $800 \mathrm{~nm}$ and an intensity of $0.5 \times 10^{15} \mathrm{~W} / \mathrm{cm}^{2}$. The general trends are the same for the two harmonic frequencies and also for the two isotopes. In more detail, the initial vibrational states with quantum numbers $j=0,1$ lead to very similar results. A significant drop in intensity is found for the quantum number $j=2$. Increasing the initial vibrational excitation leads to a steady decrease of the yield with the exception of the cases $j=3$ and $j=8$. It is remarkable that the HHG yield decreases by a factor of five at $n=69$ when going from the initial quantum number $j=0$ to $j=9$. The behavior

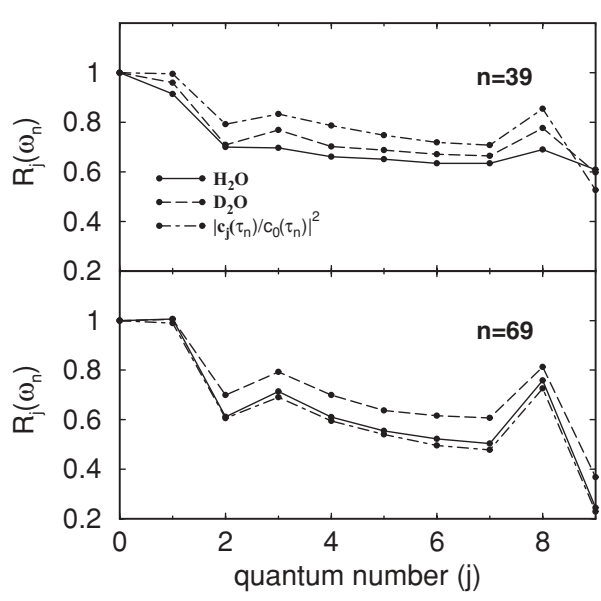

FIG. 4. Dependence of the intensity of the harmonic peak at harmonic order $n$ on the quantum number of the initial vibrational state in the neutral molecule. Shown are the ratios $R_{j}\left(\omega_{n}\right)=S_{j}\left(\omega_{n}\right) / S_{0}\left(\omega_{n}\right)$ of the signals for two values of $n$ and for the isotopes $\mathrm{H}_{2} \mathrm{O}$ and $\mathrm{D}_{2} \mathrm{O}$, as indicated. Also shown is the ratio $\left|c_{j}\left(\tau_{n}\right) / c_{0}\left(\tau_{n}\right)\right|^{2}$ of autocorrelation functions for $\mathrm{H}_{2} \mathrm{O}$ at the classical excursion time $\tau_{n}$ at which the corresponding harmonic frequency is produced.

of the ratios as a function of the initial excitation correlates directly with the autocorrelation functions at the classical electron excursion times $\tau_{n}$ at which the respective harmonic frequency $\omega_{n}$ is generated [19]. We calculate these times using the formula [19]:

$$
\tau_{n}=\frac{1}{\omega_{L}}\left\{0.786\left[f\left(x_{n}\right)\right]^{1.207}+3.304\left[f\left(x_{n}\right)\right]^{0.492}\right\}
$$

with

$$
f\left(x_{n}\right)=\frac{1}{\pi} \arccos \left(1-\frac{x_{n}}{1.5866}\right), \quad x_{n}=\frac{E_{n}}{U_{p}} .
$$

Here enters the laser frequency $\omega_{L}$ and the return energy of the electron, $E_{n}=\omega_{n}-I_{p}$. The ponderomotive potential is $U_{p}=\left(\tilde{E}_{0}^{2}\right) /\left(4 \omega_{L}^{2}\right)$, where $\tilde{E}_{0}$ is the peak intensity of the laser field. The latter equations take only the shortest trajectories into account. This is reasonable in our case because the autocorrelation function decreases rapidly for times larger than $2 \mathrm{fs}$, so that trajectories with longer travel times are quenched. Equation (15) yields the times $\tau_{39}=1.15 \mathrm{fs}$ and $\tau_{69}=1.69 \mathrm{fs}$, respectively. Figure 4 contains the ratio $\left|c_{j}\left(\tau_{n}\right) / c_{0}\left(\tau_{n}\right)\right|^{2}$ calculated for $\mathrm{H}_{2} \mathrm{O}$. It is obvious that the overall dependence of the intensity ratios on the vibrational quantum number follows the values of the ratio of the respective autocorrelation functions. Figure 4 shows that, in contrast to HHG from the vibronic ground state, for vibrationally excited states the nuclear dynamics is of importance and cannot be ignored. Similar to studies of methane [20] and molecular hydrogen [19], the harmonic emission is usually larger for the heavier isotope.

To make this more quantitative, in Fig. 5 we show the ratio $\sigma_{j}^{\mathrm{D}}\left(\omega_{n}\right) / \sigma_{j}^{\mathrm{H}}\left(\omega_{n}\right)$ between the (integrated) harmonic peaks, where the superscripts $\mathrm{H}$ and $\mathrm{D}$ identify the two isotopes. Again, we show results for the harmonic frequencies $\omega_{39}$ and $\omega_{69}$. For the lower harmonic, the isotope effect is quite small, whereas in the case of $n=69$, ratios up to 1.6 are found. Although this is still not dramatic, it shows that the 


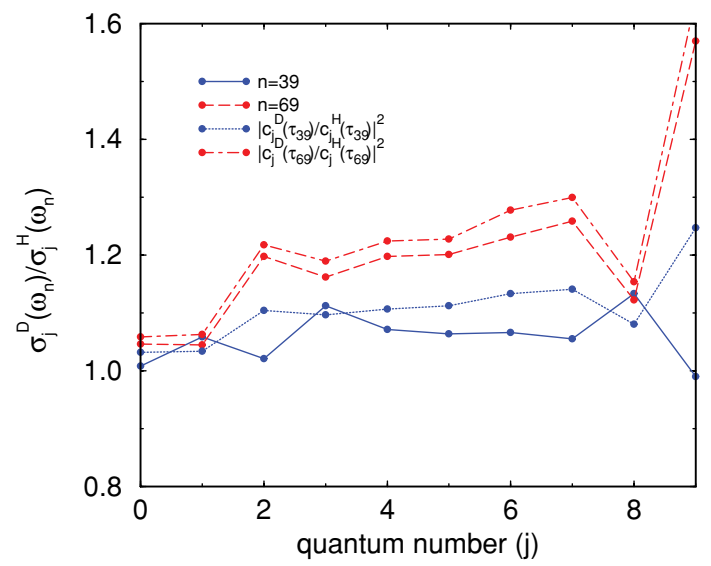

FIG. 5. (Color online) Ratio of harmonic signals from $\mathrm{D}_{2} \mathrm{O}$ and $\mathrm{H}_{2} \mathrm{O}$ as a function of the quantum number of the initial vibrational state in the neutral molecule. Shown are the ratios of integrated harmonic peaks for two different harmonic orders $n$ (solid blue and dashed red curves) as well as the corresponding ratios expected from the autocorrelation function (dotted blue and dot-dashed red curves).

nuclear dynamics for the vibrationally excited states induces visible deviations between the molecules $\mathrm{H}_{2} \mathrm{O}$ and $\mathrm{D}_{2} \mathrm{O}$. Also shown in the figure is the comparison with the ratios $\left|c_{j}^{\mathrm{D}}\left(\tau_{n}\right) / c_{j}^{\mathrm{H}}\left(\tau_{n}\right)\right|^{2}$ obtained from the autocorrelation functions. This confirms that the isotope effect due to vibrational motion is more pronounced for higher harmonic orders. It is clear that longer recombination times (corresponding to higher harmonic orders) imply a larger difference in the vibrational motions in the ionic state.

To conclude, we investigate high-order harmonic generation in water molecules within the SFA. It is shown that no isotope effect is present if the neutral molecules are initially in their vibrational ground state. In this case, the nuclear motion taking place on the timescale of the radiative recombination can safely be ignored. As a consequence, harmonic spectra for $\mathrm{H}_{2} \mathrm{O}$ and $\mathrm{D}_{2} \mathrm{O}$ are almost identical.

Regarding molecules in vibrationally excited initial states, this picture changes. First, the intensities of the harmonic peaks depend on the degree of initial vibrational excitation and decrease by factors up to 5 when compared to HHG starting from the ground state of the neutral molecule. Second, a clear isotope effect is encountered. It is more pronounced for larger vibrational quantum numbers of the initial state. For the range of initial states that we have investigated, factors around 1.2 are encountered with a maximum value of about 1.6.

Our findings are consistent with the conclusions of Ref. [35]. There it was shown that the autocorrelation functions for excited states of the NO molecule decay much faster than for the ground state, without calculating the harmonic spectra. This phenomenon requires further analysis because it may complicate the interpretation of pump-probe measurements of nuclear dynamics [16,17]. Until now, this pump-probe scheme rested on the assumption that the generation of harmonics can be used as a probe process during which the nuclear motion is negligible.

\section{ACKNOWLEDGMENTS}

MF acknowledges financial support by the State of Bavaria (Bayerisches Eliteförderungsgesetz). ML thanks the Deutsche Forschungsgemeinschaft for the funding for the Centre for Quantum Engineering and Space-Time Research (QUEST). We thank R. Fink for stimulating discussions.
[1] A. McPherson et al., J. Opt. Soc. Am. B 4, 595 (1987).

[2] A. L'Huillier, K. J. Schafer, and K. C. Kulander, J. Phys. B 24, 3315 (1991).

[3] G. Sansone et al., Science 314, 443 (2006).

[4] E. Goulielmakis et al., Science 320, 1614 (2008).

[5] R. Velotta, N. Hay, M. B. Mason, M. Castillejo, and J. P. Marangos, Phys. Rev. Lett. 87, 183901 (2001).

[6] R. de Nalda, E. Heesel, M. Lein, N. Hay, R. Velotta, E. Springate, M. Castillejo, and J. P. Marangos, Phys. Rev. A 69, 031804(R) (2004).

[7] J. Itatani, D. Zeidler, J. Levesque, M. Spanner, D. M. Villeneuve, and P. B. Corkum, Phys. Rev. Lett. 94, 123902 (2005).

[8] M. Lein, N. Hay, R. Velotta, J. P. Marangos, and P. L. Knight, Phys. Rev. Lett. 88, 183903 (2002).

[9] M. Lein, N. Hay, R. Velotta, J. P. Marangos, and P. L. Knight, Phys. Rev. A 66, 023805 (2002).

[10] T. Kanai, S. Minemoto, and H. Sakai, Nature (London) 435, 470 (2005).

[11] C. Vozzi et al., Phys. Rev. Lett. 95, 153902 (2005).

[12] O. Smirnova et al., Nature (London) 460, 972 (2009).

[13] B. K. McFarland, J. P. Farrell, P. H. Bucksbaum, and M. Gühr, Science 322, 1232 (2008).
[14] R. Torres et al., Phys. Rev. Lett. 98, 203007 (2007).

[15] J. Itatani et al., Nature (London) 432, 867 (2004).

[16] N. Wagner et al., Proc. Nat. Acad. Sci. USA 103, 13279 (2006).

[17] W. Li et al., Science 322, 1207 (2008).

[18] P. B. Corkum, Phys. Rev. Lett. 71, 1994 (1993).

[19] M. Lein, Phys. Rev. Lett. 94, 053004 (2005).

[20] S. Baker et al., Science 312, 424 (2006).

[21] S. Baker et al., Phys. Rev. Lett. 101, 053901 (2008).

[22] A. Flettner, T. Pfeifer, D. Walter, C. Winterfeldt, C. Spielmann, and G. Gerber, Appl. Phys. B 77, 747 (2003).

[23] P. Andresen, V. Beushausen, D. Häusler, H. W. Lülf, and E. W. Rothe, J. Chem. Phys. 83, 1429 (1985).

[24] V. Engel et al., J. Phys. Chem. 96, 3201 (1992).

[25] R. L. Vander Wal and F. F. Crim, J. Phys. Chem. 93, 5331 (1989).

[26] R. L. Vander Wal, J. L. Scott, and F. F. Crim, J. Chem. Phys. 94, 1859 (1991).

[27] W. Jakubetz, J. Manz, and V. Mohan, J. Chem. Phys. 90, 3686 (1989).

[28] S. Chelkowski, T. Zuo, O. Atabek, and A. D. Bandrauk, Phys. Rev. A 52, 2977 (1995).

[29] K. C. Kulander, F. H. Mies, and K. J. Schafer, Phys. Rev. A 53, 2562 (1996). 
[30] W. Qu, Z. Chen, Z. Xu, and C. H. Keitel, Phys. Rev. A 65, 013402 (2001)

[31] B. Feuerstein and U. Thumm, Phys. Rev. A 67, 043405 (2003).

[32] T. Kreibich, M. Lein, V. Engel, and E. K. U. Gross, Phys. Rev. Lett. 87, 103901 (2001).

[33] S. Saugout, C. Cornaggia, A. Suzor-Weiner, and E. Charron, Phys. Rev. Lett. 98, 253003 (2007).

[34] M. Lewenstein, P. Balcou, M. Y. Ivanov, A. L'Huillier, and P. B. Corkum, Phys. Rev. A 49, 2117 (1994).

[35] S. Patchkovskii, Phys. Rev. Lett. 102, 253602 (2009).

[36] C. C. Chirilă and M. Lein, J. Phys. B 39, S437 (2006).
[37] L. Karlsson et al., J. Chem. Phys. 62, 4745 (1975).

[38] W. Kutzelnigg, Einführung in die Theoretische Chemie, Band 2 (VCH, Weinheim, Germany, 1994).

[39] J. C. Slater, Phys. Rev. 36, 57 (1930).

[40] B. Weis, S. Carter, P. Rosmus, H.-J. Werner, and P. J. Knowles, J. Chem. Phys. 91, 2818 (1989).

[41] J. R. Reimers and R. O. Watts, Mol. Phys. 52, 357 (1984).

[42] R. Kosloff and H. Tal-Ezer, Chem. Phys. Lett. 127, 223 (1986).

[43] M. D. Feit, J. A. Fleck, and A. Steiger, J. Comput. Phys. 47, 412 (1982). 